\title{
Neutrosophic ratio-type estimators for estimating the population mean
}

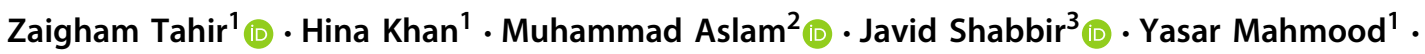 \\ Florentin Smarandache ${ }^{4}$
}

Received: 16 March 2021 / Accepted: 10 June 2021 / Published online: 9 August 2021

(c) The Author(s) 2021

\begin{abstract}
All researches, under classical statistics, are based on determinate, crisp data to estimate the mean of the population when auxiliary information is available. Such estimates often are biased. The goal is to find the best estimates for the unknown value of the population mean with minimum mean square error (MSE). The neutrosophic statistics, generalization of classical statistics tackles vague, indeterminate, uncertain information. Thus, for the first time under neutrosophic statistics, to overcome the issues of estimation of the population mean of neutrosophic data, we have developed the neutrosophic ratio-type estimators for estimating the mean of the finite population utilizing auxiliary information. The neutrosophic observation is of the form $Z_{N}=Z_{L}+Z_{U} I_{N}$ where $I_{N} \in\left[I_{L}, I_{U}\right], Z_{N} \in\left[Z_{l}, Z_{u}\right]$. The proposed estimators are very helpful to compute results when dealing with ambiguous, vague, and neutrosophic-type data. The results of these estimators are not single-valued but provide an interval form in which our population parameter may have more chance to lie. It increases the efficiency of the estimators, since we have an estimated interval that contains the unknown value of the population mean provided a minimum MSE. The efficiency of the proposed neutrosophic ratio-type estimators is also discussed using neutrosophic data of temperature and also by using simulation. A comparison is also conducted to illustrate the usefulness of Neutrosophic Ratio-type estimators over the classical estimators.
\end{abstract}

Keywords Neutrosophic $\cdot$ Classical statistics $\cdot$ Ratio estimators $\cdot$ Bias $\cdot$ Mean square error

\section{Introduction}

Hina Khan

hinakhan@gcu.edu.pk

Zaigham Tahir

zghmscholar@gmail.com

Muhammad Aslam

aslam_ravian@hotmail.com

Javid Shabbir

js@qau.edu.pk

Yasar Mahmood

syed.yasar@gcu.edu.pk

Florentin Smarandache

fsmarandache@gmail.com

1 Department of Statistics, Government College University, Lahore, Pakistan

2 Department of Statistics, Faculty of Science, King Abdulaziz University, Jeddah 21551, Saudi Arabia

3 Department of Statistics, Quaid-i-Azam University, Islamabad, Pakistan
Data in classical statistics are known and formed by crisp numbers. Many authors worked on several estimators for estimating the mean of the finite population in the existence of auxiliary information under classical statistics. "The study suggested that in the presence of high correlation between the study variable and auxiliary variable, we get significantly low sampling error for ratio, instead of taking the study variable only and hence, we may need less sampling for ratio estimation method or the ratio estimation method reduces the sample size providing equal precision [13]". A detailed discussion on ratio estimation and its properties and examples were present in one study ([14], pp. 150-186). Furthermore, one study discussed the applications of a ratio-type estimator for multivariate k-statistics [23]. More studies and various uses and types of ratio-type estimation techniques devel-

4 University of New Mexico, 705 Gurley Ave, Gallup, NM 87301, USA 
oped as time passed. The use of auxiliary information with the coefficient of variation was also studied $[22,28]$. The known parameters or other known statistics were used as auxiliary variables by various researchers $[18,24,25]$. The transformations of the auxiliary variables were also studied [31]. The performance of ratio-type estimators was improved when using different types of auxiliary information [24]. One suggested an exponential-type of ratio estimation [8]; others try to improve the exponential-type ratio estimators [32]. One studied the estimation of mean by exponential ratio-type estimators in the presence of non-response [24]. A study proposed that their estimator using complete information is a better version of the exponential ratio-type estimator [19].

"Classical statistics deal with determined data when there is no uncertainty in measurements of the observations. Therefore, we need new methods to deal with the data which are not determined. The fuzzy logic is one solution to tackle data, where we might not have exact measurements of the variable under study. Fuzzy statistics are used to analyze the data having fuzzy, ambiguous, uncertain, or imprecise parameters/ observations, but it ignores the measure of indeterminacy. Whereas, neutrosophic logic is characterized as the generalization of fuzzy logic, and it allows to measure indeterminacy along with determinate part of the observations and used to analyze under vague/ uncertain observations [2,3]."

Methods under fuzzy logic are being developed rapidly and used widely in the decision-making environment $[1,17]$. Further advancement in the fuzzy sets is complex fuzzy sets, and its generalized form is a complex neutrosophic set [21]. A study provided a detailed flow chart of fuzzy sets and their generalizations, along with a discussion on some properties and operations, including interval-valued neutrosophic sets [20].

In decision-making problems, if the fuzzy set fails to handle uncertainty, then the neutrosophic set is a better alternative. Neutrosophic sets are classified into many types. One study presented a trapezoidal bipolar neutrosophic number and its classification for decision-making problems [11]. One study introduced generalized spherical fuzzy numbers and established a detailed analysis scheme and more related methods for multi-criteria group decision making (MCGDM) [16]. Another study suggested arithmetic and geometric operations under pentagonal neutrosophic numbers along with the application based on MCGDM in mobile communication [9]. The neutrosophic numbers are gaining much interest of the researchers as time passes, for instance, an MCGDM scheme was proposed under the cylindrical neutrosophic domain [10].

Thus, in problems when the data have some indeterminacy, neutrosophic statistics are used. Neutrosophic statistics is an extension of classical statistics, used when there is neutrosophy in data or a sample. When observations in the population or the sample are imprecise, indeterminate, and vague, then neutrosophic statistics are applied [29].

\section{Neutrosophic data}

Neutrosophic data refer to a data set that is indeterminate to some degree, and neutrosophic statistical methods are used to analyze such data. The sample size, in neutrosophic statistics, may not be known as the exact number [29]. Researchers discussed that neutrosophic statistics are very effective and suitable for applying them in the uncertainty system $[2,30]$. In rock engineering, to study the scale effect and anisotropy of joint roughness coefficient, Neutrosophic numbers had been used, which results in a better and effective method to overcome the loss in information giving sufficient fitted functions [12]. New neutrosophic analysis of variance technique presented under neutrosophic data [3]. The area of neutrosophic interval statistics (NIS), neutrosophic applied statistics (NAS), and neutrosophic statistical quality control (NSQC) were developed by [4-7].

\section{Research gap}

All previous researches on survey sampling are on the type of data that are determined, certain, and clear. These methods provide a single crisp result, which may have chances of being wrong, over, or underestimated, which is a drawback sometimes. However, in many cases, data are of neutrosophic nature under some circumstances; this is the point, where Neutrosophic statistics is applied, and old classical methods failed. Data of neutrosophic nature are uncertain and ambiguous observations, non-clear arguments, and vague interval values. Thus, the information obtained from experiments or populations may behave as interval-valued neutrosophic numbers (INN). The actual observation, which is indeterminate at the time of collection, was believed to be a value that belongs to that interval. In real life, more indeterminate data are available than the determinate data. Therefore, more neutrosophic statistical techniques are required.

In life, many study variables are available for whom the collection of information is very expensive, especially when the information is ambiguous. Therefore, it will be risky and costly to compute the unknown true value of the population by the old classical methods for indeterminate data. When the study variable and auxiliary variables are of neutrosophic nature, there is no method available to solve the problem using ratio estimation. Thus, a neutrosophic ratio-type estimation method is proposed in this study.

After exhaustive research of the published studies, no research has been found in survey sampling for the ratiotype estimation methods to estimate the unknown population mean in the presence of auxiliary variables under neutro- 
sophic data. This field of statistics is yet to be filled with promising articles. This study is the first step in this area.

\section{Scope of the study}

Neutrosophic Statistical analysis helps deal with the data containing a certain amount of indeterminacy or incomplete information. In addition, this method allows for inconsistent beliefs as well. Data collection through some tools might present some observations in a range of uncertain values with the chance of inclusion of an actual measurement in that range. In the case of indeterminacy, classical statistics failed to analyze data. Hence, neutrosophic statistics is applied under the uncertain environment, which is the alternative and generalization of Classical Statistics and more flexible. Considerable researches have been done so far in the field of survey sampling under the Neutrosophy, in which the ratio estimation is still fresh and requires a great deed of attention for the uncertain system of data. For example, if we take measurements of a machine's product (say produces nuts or bolts), it might manufacture items with minor measurement errors or manufacturing errors, and we can accept that product if it lies in the particular range of measurement. In these cases, if we use classical statistics providing a single-valued result will cause lots of loss by rejecting the items even these are usable. Thus, neutrosophic statistics can cover these problems by providing the best estimate of interval results with the least MSE.

\section{Neutrosophic observation}

Several types of neutrosophic observations, including quantitative neutrosophic data, were presented, which stated that a number might lie in the interval $[a, b]$ (unknown exactly). [30]. The interval value of neutrosophic numbers can be exhibit in many ways. We have taken neutrosophic interval values as $Z_{N}=Z_{L}+Z_{U} I_{N}$ with $I_{N} \in\left[I_{L}, I_{U}\right]$. Thus, we used notation for our neutrosophic data, which are in the interval form $Z_{N} \epsilon[a, b]$, where ' $a$ ' is lower value and ' $b$ ' is the upper value of the neutrosophic data.

First, this study proposed several neutrosophic estimators for estimating the mean of the finite population in the presence of auxiliary information, which is very suitable to overcome the problem of sample indeterminacy.

\section{Terminology}

Consider a neutrosophic random sample of size $n_{N} \in$ $\left[n_{L}, n_{U}\right]$, which is drawn from a finite population of ' $N$ ' units $\left(T_{1}, T_{2}, \ldots, T_{N}\right)$. Let $y_{N}(i)$ is the ith sample obser- vation of our neutrosophic data, which is of the form $y_{N}$ (i) $\in\left[y_{L}, y_{U}\right]$ and similarly for auxiliary variable $x_{N}$ (i) $\in\left[x_{L}, x_{U}\right]$. Let $\bar{y}_{N}(i) \in\left[\bar{y}_{L}, \bar{y}_{u}\right]$ is our neutrosophic variable of interest, and $\bar{x}_{N}(i) \in\left[\bar{x}_{L}, \bar{x}_{U}\right]$ is our auxiliary neutrosophic variable which is correlated to our study variable $\bar{y}_{N}$. In addition, $Y_{N} \in\left[Y_{L}, Y_{U}\right]$ and $X_{N} \in\left[X_{L}\right.$, $\left.X_{U}\right]$ are the overall averages of the neutrosophic set of data. $C_{y N} \in\left[C_{y N L}, C_{y N U}\right]$ and $C_{x N} \in\left[C_{x N L}, C_{x N U}\right]$ are neutrosophic coefficients of variation for $Y_{N}$ and $X_{N}$, respectively. $\rho_{x y N}$ is the neutrosophic correlation between $X_{N}$ and $Y_{N}$ (neutrosophic variables). In addition, $\beta_{2(x) N} \in\left[\beta_{2(x) L}\right.$, $\left.\beta_{2(x) U}\right]$ is the neutrosophic coefficient of kurtosis for auxiliary variable $X_{N} \cdot \bar{e}_{y N} \in\left[\bar{e}_{y L}, \bar{e}_{y U}\right]$ and $\bar{e}_{x N} \in\left[\bar{e}_{x L}, \bar{e}_{y U}\right]$ are the neutrosophic mean errors. These terms can be computed by the following relations. Similarly, $\operatorname{Bias}\left(\bar{y}_{N}\right) \in\left[\operatorname{Bias}_{L}\right.$, Bias $\left._{U}\right]$ and MSE, $M S E\left(\bar{y}_{N}\right) \in\left[M S E_{L}, M S E_{U}\right]$ belong to the neutrosophic sets were also computed for the analysis: $\bar{e}_{y N}(i)=\bar{y}_{N}(i)-\bar{Y}_{N} ; \bar{e}_{x N}(i)=\bar{x}_{N}(i)-\bar{X}_{N}$;

$$
E\left(\bar{e}_{y N}\right)=E\left(\bar{e}_{x N}\right)=0
$$

$\mathrm{E}\left(\bar{e}_{y N}^{2}\right)=\theta_{N} \bar{Y}_{N}^{2} C_{y N}^{2} ; \quad E\left(\bar{e}_{x N}^{2}\right)=\theta_{N} \bar{X}_{N}^{2} C_{x N}^{2}$

$E\left(\bar{e}_{y N} \bar{e}_{x N}\right)=\theta_{N} \bar{X}_{N} \bar{Y}_{N} C_{x N} C_{y N} \rho_{x y N}$

where $\bar{e}_{y N} \in\left[\bar{e}_{y L}, \bar{e}_{y U}\right] ; \bar{e}_{x N} \in\left[\bar{e}_{x L}, \bar{e}_{x U}\right] ; e_{y N} e_{x N} \in$ $\left[\bar{e}_{x L} \bar{e}_{y L}, \bar{e}_{x U} \bar{e}_{y U}\right]$

$e_{y N}^{2} \in\left[e_{y L}^{2}, e_{y U}^{2}\right] ; e_{x N}^{2} \in\left[e_{x L}^{2}, e_{x U}^{2}\right]$

$C_{x N}^{2}=\frac{\sigma_{x N}^{2}}{\bar{X}_{N}^{2}} ; C_{x N}^{2} \in\left[C_{x L}^{2}, C_{x U}^{2}\right] ; C_{y N}^{2}$ $=\frac{\sigma_{y N}^{2}}{\bar{Y}_{N}^{2}} ; C_{y N}^{2} \in\left[C_{y L}^{2}, C_{y U}^{2}\right]$

$\rho_{x y N}=\frac{\sigma_{x y N}}{\sigma_{x N} \sigma_{y N}} ; \rho_{x y N} \in\left[\rho_{x y L}, \rho_{x y U}\right]$

$\therefore \theta_{N}=\frac{1-f_{N}}{n_{N}} ; \theta_{N} \in\left[\theta_{L}, \theta_{U}\right] ; n_{N} \in\left[n_{L}, n_{U}\right]$

$\sigma_{x N}^{2} \in\left[\sigma_{x L}^{2}, \sigma_{x U}^{2}\right] ; \sigma_{y N}^{2} \in\left[\sigma_{y L}^{2}, \sigma_{y U}^{2}\right] ; \sigma_{x y N} \in\left[\sigma_{x y L}, \sigma_{x y U}\right]$ 


\section{Flow chart}

The following flow chart explains the path of using proposed methods under neutrosophic numbers. where $\bar{y}_{R N} \in\left[\bar{y}_{R L}, \bar{y}_{R U}\right], \bar{y}_{N} \in\left[\bar{y}_{L}, \bar{y}_{U}\right], \bar{x}_{N} \in\left[\bar{x}_{L}, \bar{x}_{U}\right]$, $\bar{X}_{N} \in\left[\bar{X}_{L}, \bar{X}_{U}\right], \bar{Y}_{N} \in\left[\bar{Y}_{L}, \bar{Y}_{U}\right]$

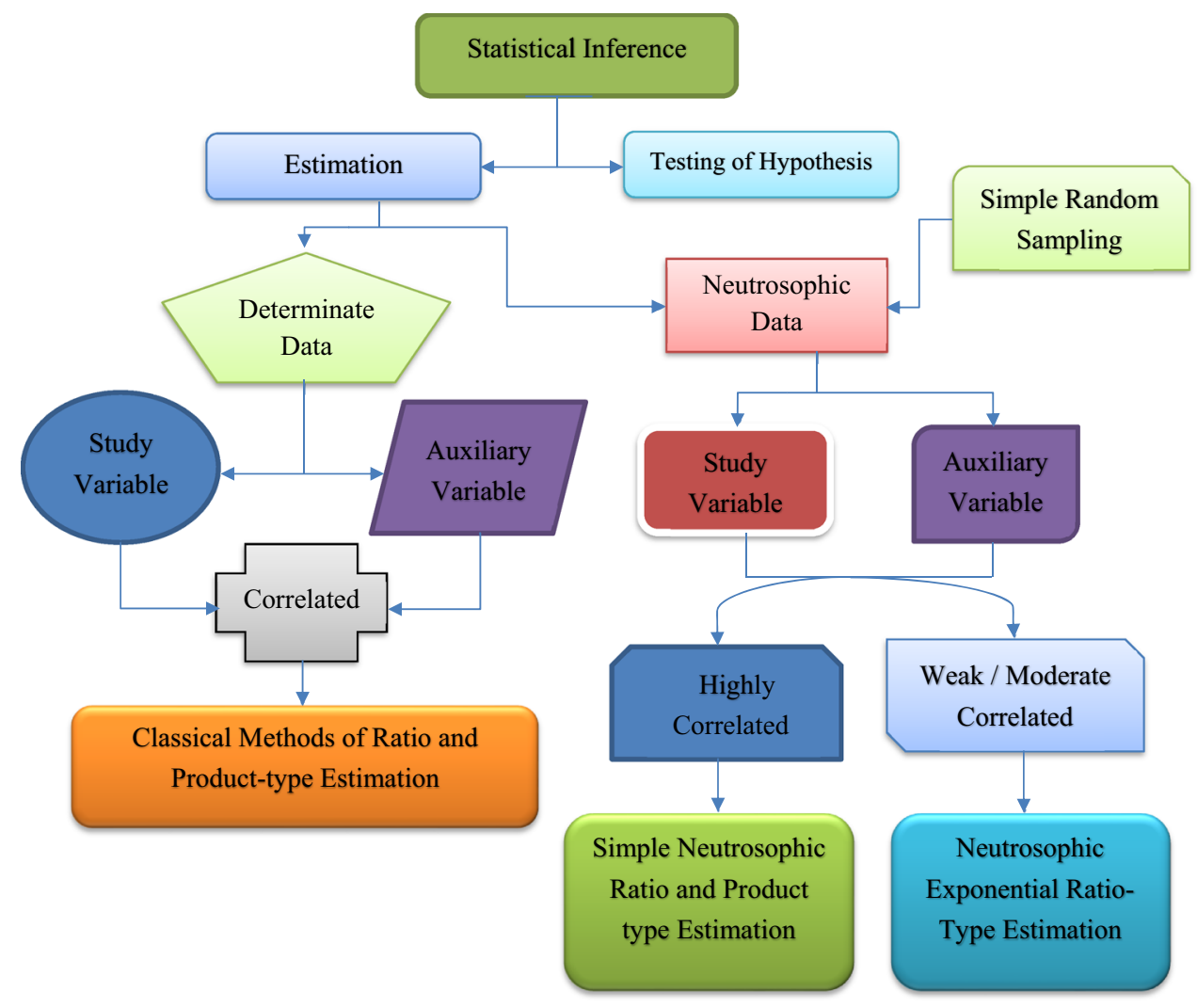

\section{Proposed neutrosophic estimators}

Here, several existing estimators were transformed into neutrosophic estimators to overcome the problem of data indeterminacy and neutrosophic data.

\section{Neutrosophic ratio estimator}

The following is a proposed neutrosophic ratio estimator for estimating the mean of the finite population in the presence of auxiliary variables:

$$
\begin{aligned}
& \bar{y}_{R N}=\frac{\bar{y}_{N}}{\bar{x}_{N}} \bar{X}_{N} \\
& \bar{y}_{R N}=\left(\bar{Y}_{N}+\bar{e}_{y N}\right)\left(1+\frac{\bar{e}_{x N}}{\bar{X}_{N}}\right)^{-1}
\end{aligned}
$$

and $\bar{e}_{y N} \in\left[\bar{e}_{y L}, \bar{e}_{y U}\right] ; \bar{e}_{x N} \in\left[\bar{e}_{x L}, \bar{e}_{x U}\right]$.

The bias and MSE of $\bar{y}_{R N}$ up to first-order approximation are given by

$\operatorname{Bias}\left(\bar{y}_{R N}\right)=\theta_{N} \bar{Y}_{N}\left[C_{x N}^{2}-C_{x N} C_{y N} \rho_{x y N}\right]$

$$
\operatorname{MSE}\left(\bar{y}_{R N}\right)=\theta_{N} \bar{Y}_{N}^{2}\left[C_{y N}^{2}+C_{x N}^{2}-2 C_{x N} C_{y N} \rho_{x y N}\right]
$$

where $\theta_{N} \in\left[\theta_{L}, \theta_{U}\right] ; n_{N} \in\left[n_{L}, n_{U}\right]$

$$
\begin{aligned}
& C_{x N}^{2} \in\left[C_{x L}^{2}, C_{x U}^{2}\right] \\
& C_{y N}^{2} \in\left[C_{y L}^{2}, C_{y U}^{2}\right], \rho_{x y N} \in\left[\rho_{x y L}, \rho_{x y U}\right]
\end{aligned}
$$




\section{Several modified neutrosophic ratio estimators}

Motivated by [28], we have developed a modified neutrosophic ratio estimator, where we used the coefficient of variation as an auxiliary variable:

$\bar{y}_{S D r N}=\bar{y}_{N} \frac{\bar{X}_{N}+C_{x N}}{\bar{x}_{N}+C_{x N}}$

$\bar{y}_{S D r N}=\left(\bar{Y}_{N}+\bar{e}_{y N}\right)\left(1+\frac{\bar{e}_{x N}}{\bar{X}_{N}+C_{x N}}\right)^{-1}$

where $\bar{y}_{S D r N} \in\left[\bar{y}_{S D r L}, \bar{y}_{S D r U}\right], \bar{y}_{N} \in\left[\bar{y}_{L}, \bar{y}_{U}\right], \bar{x}_{N} \in$ $\left[\bar{x}_{L}, \bar{x}_{U}\right], \bar{X}_{N} \in\left[\bar{X}_{L}, \bar{X}_{U}\right], \bar{Y}_{N} \in\left[\bar{Y}_{L}, \bar{Y}_{U}\right]$ and $\bar{e}_{y N} \in$ $\left[\bar{e}_{y L}, \bar{e}_{y U}\right] ; \bar{e}_{x N} \in\left[\bar{e}_{x L}, \bar{e}_{x U}\right], C_{x N} \in\left[C_{x L}, C_{x U}\right]$

Expressions of bias and MSE of $\bar{y}_{S D r N}$ up to first-order approximation are given as

$\operatorname{Bias}\left(\bar{y}_{S D r N}\right)$

$$
=\theta_{N} \bar{Y}_{N}\left[\left(\frac{\bar{X}_{N}}{\bar{X}_{N}+C_{x N}}\right)^{2} C_{x N}^{2}-\left(\frac{\bar{X}_{N}}{\bar{X}_{N}+C_{x N}}\right) C_{x N} C_{y N} \rho_{x y N}\right]
$$

$\operatorname{MSE}\left(\bar{y}_{S D r N}\right)$

$$
=\theta_{N} \bar{Y}_{N}^{2}\left[C_{y N}^{2}+\left(\frac{\bar{X}_{N}}{\bar{X}_{N}+C_{x N}}\right)^{2} C_{x N}^{2}-2\left(\frac{\bar{X}_{N}}{\bar{X}_{N}+C_{x N}}\right) C_{x N} C_{y N} \rho_{x y N}\right]
$$

where $\theta_{N} \in\left[\theta_{L}, \theta_{U}\right] ; n_{N} \in\left[n_{L}, n_{U}\right]$

$$
\begin{aligned}
C_{x N}^{2} & \in\left[C_{x L}^{2}, C_{x U}^{2}\right], \\
C_{y N}^{2} & \in\left[C_{y L}^{2}, C_{y U}^{2}\right], \rho_{x y N} \in\left[\rho_{x y L}, \rho_{x y U}\right]
\end{aligned}
$$

Now, another neutrosophic estimator is suggested, where we have considered the coefficient of kurtosis as an auxiliary variable:

$\bar{y}_{S K r N}=\bar{y}_{N} \frac{\bar{X}_{N}+\beta_{2(x) N}}{\bar{x}_{N}+\beta_{2(x) N}}$

$\bar{y}_{S K r N}=\left(\bar{Y}_{N}+\bar{e}_{y N}\right)\left(1+\frac{\bar{e}_{x N}}{\bar{X}_{N}+\beta_{2(x) N}}\right)^{-1}$

where $\bar{y}_{S K r N} \in\left[\bar{y}_{S K r L}, \bar{y}_{S K r U}\right], \bar{y}_{N} \in\left[\bar{y}_{L}, \bar{y}_{U}\right], \bar{x}_{N} \in$ $\left[\bar{x}_{L}, \bar{x}_{U}\right], \bar{X}_{N} \in\left[\bar{X}_{L}, \bar{X}_{U}\right], \bar{Y}_{N} \in\left[\bar{Y}_{L}, \bar{Y}_{U}\right]$ and $\beta_{2(x) N} \in$ $\left[\beta_{2(x) L}, \beta_{2(x) U}\right], \bar{e}_{y N} \in\left[\bar{e}_{y L}, \bar{e}_{y U}\right], \bar{e}_{x N} \in\left[\bar{e}_{x L}, \bar{e}_{x U}\right]$
The bias and MSE of $\bar{y}_{S K r N}$ correct up to first-order approximation are given by

$$
\begin{aligned}
\operatorname{Bias}\left(\bar{y}_{S K r N}\right)= & \theta_{N} \bar{Y}_{N}\left[\left(\frac{\bar{X}_{N}}{\bar{X}_{N}+\beta_{2(x) N}}\right)^{2} C_{x N}^{2}\right. \\
& \left.-\left(\frac{\bar{X}_{N}}{\bar{X}_{N}+\beta_{2(x) N}}\right) C_{x N} C_{y N} \rho_{x y N}\right] \\
\operatorname{MSE}\left(\bar{y}_{S K r N}\right)= & \theta_{N} \bar{Y}_{N}^{2}\left[C_{y N}^{2}+\left(\frac{\bar{X}_{N}}{\bar{X}_{N}+\beta_{2(x) N}}\right)^{2} C_{x N}^{2}\right. \\
& \left.-2\left(\frac{\bar{X}_{N}}{\bar{X}_{N}+\beta_{2(x) N}}\right) C_{x N} C_{y N} \rho_{x y N}\right]
\end{aligned}
$$

Motivated by [31], using both coefficient of variation and kurtosis in neutrosophic ratio-type estimator given as

$\bar{y}_{U S r N}=\bar{y}_{N} \frac{\bar{X}_{N} \beta_{2(x) N}+C_{x N}}{\bar{x}_{N} \beta_{2(x) N}+C_{x N}}$

$\bar{y}_{U S r N}=\left(\bar{Y}_{N}+\bar{e}_{y N}\right)\left(1+\frac{\beta_{2(x) N} \bar{e}_{x N}}{\bar{X}_{N} \beta_{2(x) N}+C_{x N}}\right)^{-1}$

Where $\bar{y}_{U S r N} \in\left[\bar{y}_{U S r L}, \bar{y}_{U S r U}\right], \bar{y}_{N} \in\left[\bar{y}_{L}, \bar{y}_{U}\right], \bar{x}_{N} \in$ $\left[\bar{x}_{L}, \bar{x}_{U}\right], \bar{X}_{N} \in\left[\bar{X}_{L}, \bar{X}_{U}\right], \bar{Y}_{N} \in\left[\bar{Y}_{L}, \bar{Y}_{U}\right]$ and $\beta_{2(x) N} \in$ $\left[\beta_{2(x) L}, \beta_{2(x) U}\right], C_{x N} \in\left[C_{x L}, C_{x U}\right], \bar{e}_{y N} \in\left[\bar{e}_{y L}, \bar{e}_{y U}\right]$, $\bar{e}_{x N} \in\left[\bar{e}_{x L}, \bar{e}_{x U}\right]$

The bias and MSE of $\bar{y}_{U S r N}$ to the first order of approximation are given by

$\begin{aligned} \operatorname{Bias}\left(\bar{y}_{U S r N}\right)= & \theta_{N} \bar{Y}_{N}\left[\left(\frac{\bar{X}_{N} \beta_{2(x) N}}{\bar{X}_{N} \beta_{2(x) N}+C_{x N}}\right)^{2} C_{x N}^{2}\right. \\ & \left.-\left(\frac{\bar{X}_{N} \beta_{2(x) N}}{\bar{X}_{N} \beta_{2(x) N}+C_{x N}}\right) C_{x N} C_{y N} \rho_{x y N}\right]\end{aligned}$

$\operatorname{MSE}\left(\bar{y}_{U S r N}\right)$

$$
\begin{aligned}
& =\theta_{N} \bar{Y}_{N}^{2}\left[C_{y N}^{2}+\left(\frac{\bar{X}_{N} \beta_{2(x) N}}{\bar{X}_{N} \beta_{2(x) N}+C_{x N}}\right)^{2} C_{x N}^{2}\right. \\
& \left.-2\left(\frac{\bar{X}_{N} \beta_{2(x) N}}{\bar{X}_{N} \beta_{2(x) N}+C_{x N}}\right) C_{x N} C_{y N} \rho_{x y N}\right]
\end{aligned}
$$

where $\theta_{N} \in\left[\theta_{L}, \theta_{U}\right] ; n_{N} \in\left[n_{L}, n_{U}\right]$

$$
\begin{gathered}
C_{x N}^{2} \in\left[C_{x L}^{2}, C_{x U}^{2}\right], C_{y N}^{2} \in\left[C_{y L}^{2},\right. \\
\left.C_{y U}^{2}\right], \rho_{x y N} \in\left[\rho_{x y L}, \rho_{x y U}\right] .
\end{gathered}
$$




\section{Neutrosophic exponential estimators}

Here, a neutrosophic exponential-type estimator for estimating the mean for a finite population in the presence of auxiliary variables is suggested:

$$
\begin{aligned}
& \bar{y}_{B \operatorname{TrN}}=\bar{y}_{N} \exp \left(\frac{\bar{X}_{N}-\bar{x}_{N}}{\bar{X}_{N}+\bar{x}_{N}}\right) \\
& \bar{y}_{B \operatorname{TrN}}=\left(\bar{Y}_{N}+\bar{e}_{y N}\right) \exp \left[-\frac{\bar{e}_{x N}}{2 \bar{X}_{N}}\left(1+\frac{\bar{e}_{x N}}{2 \bar{X}_{N}}\right)^{-1}\right]
\end{aligned}
$$

where $\bar{y}_{B \operatorname{TrN}} \in\left[\bar{y}_{B \operatorname{Tr} L}, \bar{y}_{B \operatorname{Tr} U}\right], \bar{y}_{N} \in\left[\bar{y}_{L}, \bar{y}_{U}\right], \bar{x}_{N} \in$ $\left[\bar{x}_{L}, \bar{x}_{U}\right], \bar{X}_{N} \in\left[\bar{X}_{L}, \bar{X}_{U}\right], \bar{Y}_{N} \in\left[\bar{Y}_{L}, \bar{Y}_{U}\right]$ and $\bar{e}_{y N} \in$ $\left[\bar{e}_{y L}, \bar{e}_{y U}\right], \bar{e}_{x N} \in\left[\bar{e}_{x L}, \bar{e}_{x U}\right]$.

The bias and MSE of $\bar{y}_{B T r N}$ up to first-order approximation are given by

$$
\begin{aligned}
& \operatorname{Bias}\left(\bar{y}_{B T r N}\right)=\theta_{N} \bar{Y}_{N}\left[\frac{3}{8} C_{x N}^{2}-\frac{1}{2} C_{x N} C_{y N} \rho_{x y N}\right] \\
& \operatorname{MSE}\left(\bar{y}_{B T r N}\right)=\theta_{N} \bar{Y}_{N}^{2}\left[C_{y N}^{2}+\frac{1}{4} C_{x N}^{2}-C_{x N} C_{y N} \rho_{x y N}\right]
\end{aligned}
$$

$$
\begin{gathered}
\text { where } \theta_{N} \in\left[\theta_{L}, \theta_{U}\right] ; n_{N} \in\left[n_{L}, n_{U}\right] \\
C_{x N}^{2} \in\left[C_{x L}^{2}, C_{x U}^{2}\right], C_{y N}^{2} \in\left[C_{y L}^{2},\right. \\
\left.C_{y U}^{2}\right], \rho_{x y N} \in\left[\rho_{x y L}, \rho_{x y U}\right]
\end{gathered}
$$

Motivated by ([26], we have developed a new neutrosophic exponential ratio-type estimator for estimating the mean of a finite population:

$$
\begin{aligned}
& \bar{y}_{R r N}=\bar{y}_{N} \exp \left[\frac{\left(a \bar{X}_{N}+b\right)-\left(a \bar{x}_{N}+b\right)}{\left(a \bar{X}_{N}+b\right)+\left(a \bar{x}_{N}+b\right)}\right] \\
& \bar{y}_{R r N}=\left(\bar{Y}_{N}+\bar{e}_{y N}\right) \exp \left[-\frac{a e_{x N}}{2\left(a \bar{X}_{N}+b\right)}\left(1+\frac{a \bar{e}_{x N}}{2\left(a \bar{X}_{N}+b\right)}\right)^{-1}\right]
\end{aligned}
$$

where $\bar{y}_{R r N} \in\left[\bar{y}_{R r L}, \bar{y}_{R r U}\right], \bar{y}_{N} \in\left[\bar{y}_{L}, \bar{y}_{U}\right], \bar{x}_{N} \in$ $\left[\bar{x}_{L}, \bar{x}_{U}\right], \bar{X}_{N} \in\left[\bar{X}_{L}, \bar{X}_{U}\right], \bar{Y}_{N} \in\left[\bar{Y}_{L}, \bar{Y}_{U}\right]$ and $\bar{e}_{y N} \in$ $\left[\bar{e}_{y L}, \bar{e}_{y U}\right], \bar{e}_{x N} \in\left[\bar{e}_{x L}, \bar{e}_{x U}\right]$

The bias and MSE of $\bar{y}_{R r N}$ up to first-order approximation are given by

$$
\begin{aligned}
\operatorname{Bias}\left(\bar{y}_{R r N}\right)= & \theta_{N} \bar{Y}_{N}\left[\frac{3}{8}\left(\frac{\bar{X}_{N}}{a \bar{X}_{N}+b}\right)^{2} C_{x N}^{2}\right. \\
& \left.-\frac{1}{2}\left(\frac{\bar{X}_{N}}{a \bar{X}_{N}+b}\right) C_{x N} C_{y N} \rho_{x y N}\right] \\
\operatorname{MSE}\left(\bar{y}_{R r N}\right)= & \theta_{N} \bar{Y}_{N}^{2}\left[C_{y N}^{2}+\left(\frac{a \bar{X}_{N}}{2\left(a \bar{X}_{N}+b\right)}\right)^{2} C_{x N}^{2}\right. \\
& \left.-\frac{1}{2}\left(\frac{2 a \bar{X}_{N}}{a \bar{X}_{N}+b}\right) C_{x N} C_{y N} \rho_{x y N}\right]
\end{aligned}
$$

where $\theta_{N} \in\left[\theta_{L}, \theta_{U}\right], n_{N} \in\left[n_{L}, n_{U}\right]$

$C_{x N}^{2} \in\left[C_{x L}^{2}, C_{x U}^{2}\right]$,

$C_{y N}^{2} \in\left[C_{y L}^{2}, C_{y U}^{2}\right], \rho_{x y N} \in\left[\rho_{x y L}, \rho_{x y U}\right]$

\section{Neutrosophic generalized exponential-type estimator}

Motivated by [19], we have developed a neutrosophic generalized exponential-type estimator for estimating the mean of a finite population:

$\bar{y}_{\mathrm{KNN}}=\bar{y}_{N} \exp \left[\alpha\left(\frac{\bar{X}_{N}^{\frac{1}{h}}-\bar{x}_{N}^{\frac{1}{h}}}{\bar{X}_{N}^{\frac{1}{h}}+(a-1) \bar{x}_{N}^{\frac{1}{h}}}\right)\right]$

where $\alpha(-\infty<\alpha<\infty)$ and $h(h>0)$ are two real constants and assumed to be known, and the other constant $a(a \neq 0)$ is supposed to be estimated so that $\bar{y}_{N G E N}$ is optimal and MSE of $\bar{y}_{N G E N}$ is minimum:

$\bar{y}_{\mathrm{KNN}}=\left(\bar{Y}_{N}+\bar{e}_{y N}\right) \exp \left[\frac{-\alpha \bar{e}_{x N}}{a h \bar{X}_{N}}\left(1+\frac{\bar{e}_{x N}}{h \bar{X}_{N}}-\frac{\bar{e}_{x N}}{a h \bar{X}_{N}}\right)^{-1}\right]$

where $\bar{y}_{K N N} \in\left[\bar{y}_{K N L}, \bar{y}_{K N U}\right], \bar{y}_{N} \in\left[\bar{y}_{L}, \bar{y}_{U}\right], \bar{x}_{N} \in$ $\left[\bar{x}_{L}, \bar{x}_{U}\right], \bar{X}_{N} \in\left[\bar{X}_{L}, \bar{X}_{U}\right], \bar{Y}_{N} \in\left[\bar{Y}_{L}, \bar{Y}_{U}\right]$ and $\bar{e}_{y N} \in$ $\left[\bar{e}_{y L}, \bar{e}_{y U}\right], \bar{e}_{x N} \in\left[\bar{e}_{x L}, \bar{e}_{x U}\right]$

The bias and MSE of $\bar{y}_{\text {NGEN }}$, correct up to first-order approximation are given by

$\operatorname{Bias}\left(\bar{y}_{\mathrm{KNN}}\right)=\theta_{N} \bar{Y}_{N}\left[\frac{\alpha C_{x N}^{2}}{a h^{2}}-\frac{\alpha C_{x N}^{2}}{a^{2} h^{2}}+\frac{\alpha^{2} C_{x N}^{2}}{2 a^{2} h^{2}}-\frac{\alpha C_{x N} C_{y N} \rho_{x y N}}{a h}\right]$

$\operatorname{MSE}\left(\bar{y}_{\mathrm{KNN}}\right)=\theta_{N} \bar{Y}_{N}^{2}\left[C_{y N}^{2}+\frac{\alpha^{2} C_{x N}^{2}}{a^{2} h^{2}}-\frac{2 \alpha C_{x N} C_{y N} \rho_{x y N}}{a h}\right]$

where $\theta_{N} \in\left[\theta_{L}, \theta_{U}\right] ; n_{N} \in\left[n_{L}, n_{U}\right]$

$C_{x N}^{2} \in\left[C_{x L}^{2}, C_{x U}^{2}\right], C_{y N}^{2} \in\left[C_{y L}^{2}\right.$,

$$
\left.C_{y U}^{2}\right], \rho_{x y N} \in\left[\rho_{x y L}, \rho_{x y U}\right]
$$

To obtain the minimum MSE, we estimate the value of ' $a$ '. From Eq.(28), the optimum value of ' $a$ ' is given by

$\widehat{a}=\frac{\alpha C_{x N}^{2}}{h C_{x N} C_{y N} \rho_{x y N}}$

We can write the expression of minimum MSE of $\left(\bar{y}_{\mathrm{KNN}}\right)$ as follows:

$\operatorname{MSE}\left(\bar{y}_{\mathrm{KNN}}\right)_{\min }=\theta_{N} \bar{Y}_{N}^{2} C_{y N}^{2}\left(1-\rho_{x y N}^{2}\right)$ 
Table 1 Population's characteristics for single auxiliary variable

\begin{tabular}{|c|c|c|c|c|c|}
\hline \multirow[t]{3}{*}{ Parameters } & \multicolumn{5}{|c|}{ Source (data): temperature of Lahore, Punjab, Pakistan from the years 2014-2019 } \\
\hline & \multicolumn{5}{|c|}{ Population available: $N=30$ (years); sample taken: $n_{N}=[6,6]$ (years) } \\
\hline & \multirow{2}{*}{$\begin{array}{l}X \\
\text { No. of Year } \\
\bar{Y}_{\mathrm{N} .}\end{array}$} & \multicolumn{4}{|c|}{$\begin{array}{l}Y \\
\text { Average temperature (min, } \max )\end{array}$} \\
\hline $\bar{X}_{N}$ & & $C_{y \mathrm{~N}}$ & $C_{x \mathrm{~N}}$ & $\rho_{x y \mathrm{~N}}$ & $\beta_{2(x) \mathrm{N}}$ \\
\hline \multirow[t]{12}{*}[3.5,3.5]{} & {$[44,64]$} & {$[0.0345,0.0360]$} & {$[0.53,0.53]$} & {$[0.23,0.40]$} & {$[-1.2,-1.2]$} \\
\hline & {$[50,72]$} & {$[0.0424,0.0529]$} & & {$[0.14,0.18]$} & \\
\hline & {$[58,80]$} & {$[0.0424,0.0454]$} & & {$[0.17,0.43]$} & \\
\hline & {$[69,94]$} & {$[0.0291,0.0293]$} & & {$[0.55,0.60]$} & \\
\hline & {$[77,102]$} & {$[0.0187,0.0282]$} & & {$[-0.04,0.04]$} & \\
\hline & {$[81,103]$} & {$[0.0272,0.0316]$} & & {$[-0.23,-0.08]$} & \\
\hline & {$[80,95]$} & {$[0.0151,0.0171]$} & & {$[-0.60,-0.49]$} & \\
\hline & {$[80,95]$} & {$[0.0108,0.0141]$} & & {$[-0.59,-0.53]$} & \\
\hline & {$[77,94]$} & {$[0.0231,0.0254]$} & & {$[0.01,0.09]$} & \\
\hline & {$[68,90]$} & {$[0.0277,0.0338]$} & & {$[-0.39,-0.20]$} & \\
\hline & {$[55,79]$} & {$[0.0233,0.0260]$} & & {$[-0.55,0.42]$} & \\
\hline & {$[45,69]$} & {$[0.0341,0.050]$} & & {$[-0.14,-0.05]$} & \\
\hline
\end{tabular}

\section{Numerical study}

\section{Empirical study}

As it is a new concept and to the best of the authors' knowledge, no work has been done so far on the neutrosophic ratio-type estimators. Therefore, in this case, we compared the MSE of the proposed Eq. (25) neutrosophic estimator with other proposed neutrosophic estimators given in Eqs. (1), (5), (9), (13), (17), and (21) to evaluate which neutrosophic ratio-type estimator performs more efficiently. We have also computed the relative efficiencies of these estimators. In statistics, for an estimator, the minimum MSE is required to be better among the class of estimators. For the numerical study, we have considered real-life indeterminacy interval data of temperature, as the daily temperature is taken as of neutrosophic nature and varies in an interval with vague values. The one reason to take temperature as neutrosophic data is that its value diverges in an interval form, where the value considers to be mention as the reference temperature of the day may be one of the lowest or highest temperatures recorded in a day or any point between them. Data of the past 6 years is noted from the weather websites available/ published online and arranged monthwise (Temperature of Lahore, Punjab, Pakistan from the years 2014-2019) described in Table 1 [15]. This data is obtained from publicly published sources, online available for all, and therefore, no ethical approval is needed. We have taken a sample of 6 years month-wise average temperature of lowest and highest temperature during each month, and $X$ is the codding of the time from 1 to 6 (number of years).
Neutrosophic averages of lower and upper limits of the temperatures of each month of 6 years were measured, which are the neutrosophic part of the data in $Y$ corresponding to known $X$ year, where the monthwise total averages for all 6 years are taken as the neutrosophic data. The temperature is taken as neutrosophic data (Temp, $\left.y_{N} \epsilon\left[y_{L}, y_{U}\right]\right)$, corresponding to time (in years $X$ ) as the independent determinate variable. The $\bar{X}_{N} \in\left[\bar{X}_{L}, \bar{X}_{U}\right]$ is an average of 6 years for which the data are collected, so it is the same value for all lower and upper limits of the corresponding neutrosophic data. $C_{x N}$ is the coefficient of variation and $\beta_{2(x) N}$ is coefficient of kurtosis of the auxiliary variable.

In Table 2, the neutrosophic MSE for the proposed estimators are given for each month of a year. MSE is arranged as upper value and lower value in Table 2 (i.e., $\mathrm{MSE}_{N} \in$ $\left.\left[\mathrm{MSE}_{U}, \mathrm{MSE}_{L}\right]\right)$. We can see the last column of Table 2 showing $\operatorname{MSE}\left(\bar{y}_{\mathrm{KNN}}\right)$ is minimum compared to others, which means it is the most efficient estimator for available neutrosophic data.

Table 3 consists of the relative efficiencies of the proposed neutrosophic ratio estimators to $\bar{y}_{\mathrm{KNN}}$. An estimator with the lowest value, i.e., a value less than or equal to 100 in comparison to all other estimators, is considered the most efficient. Here, the estimator is given in Eq. (25), is the most efficient neutrosophic ratio estimator among all, as none of the other columns giving values less than 100 .

\section{Simulation}

For evaluating efficiencies of the proposed estimators, we used simulated neutrosophic data, such that $X_{N}$ and $Y_{N}$ Neu- 

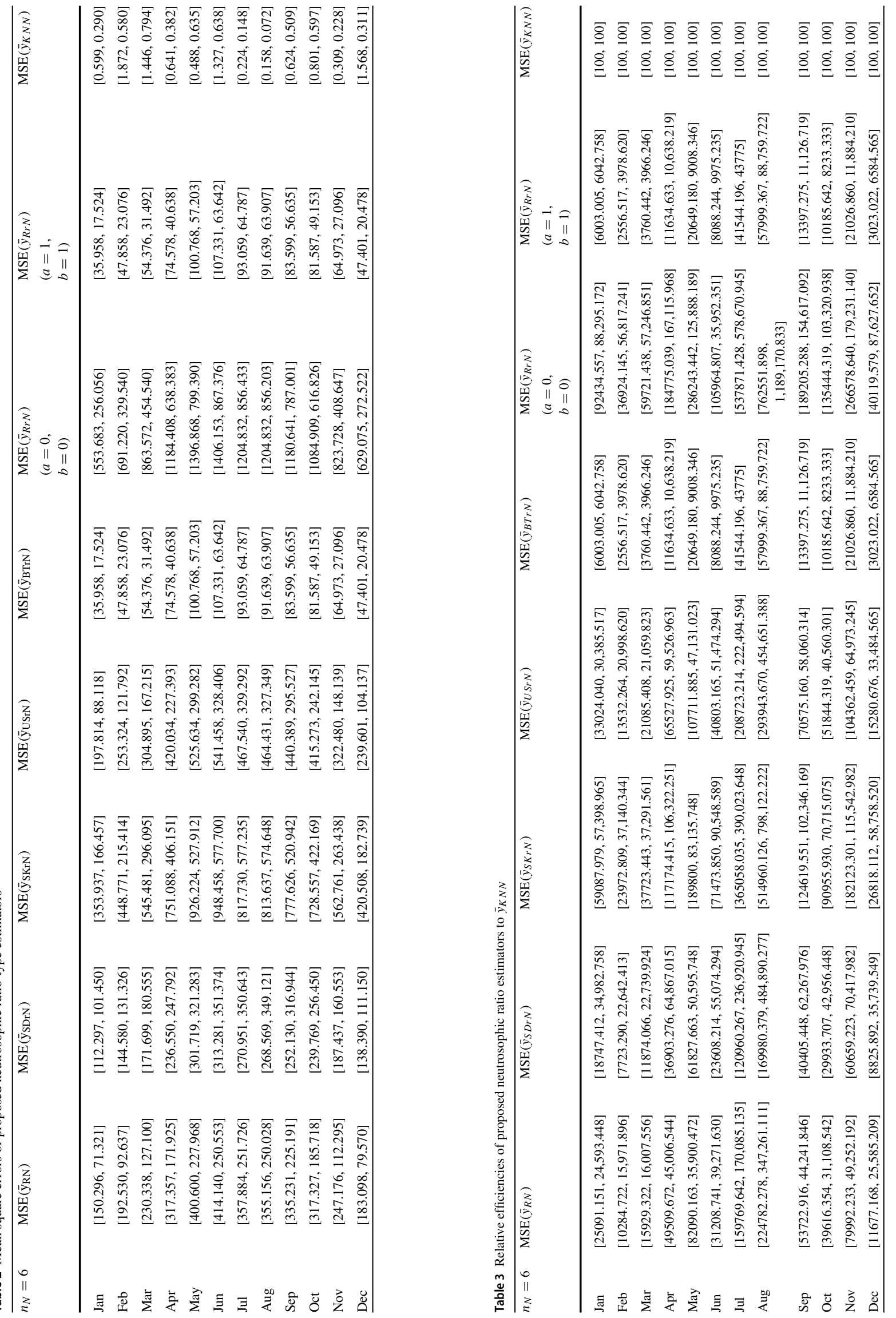
Table 4 Descriptive statistics for simulation under neutrosophic data

\begin{tabular}{llll}
\hline Parameters & $\begin{array}{l}\text { Neutrosophic } \\
\text { values }\end{array}$ & Parameters & $\begin{array}{l}\text { Neutrosophic } \\
\text { values }\end{array}$ \\
\hline$N_{N}$ & {$[1000,1000]$} & $\sigma_{y N}$ & {$[12.9,17.2]$} \\
$n_{N}$ & {$[20,20]$} & $C_{x N}$ & {$[0.03381,0.03794]$} \\
$\mu_{x N}$ & {$[171.2,180.4]$} & $C_{y N}$ & {$[0.1724,0.206]$} \\
$\mu_{y N}$ & {$[76.0,84.9]$} & $\beta_{2(x) N}$ & {$[0.2039,0.06235]$} \\
$\sigma_{x N}$ & {$[5.8,6.7]$} & $\rho_{x y N}$ & {$[0.02847,0.01041]$} \\
\hline
\end{tabular}

Table 5 Descriptive statistics for simulation under classical data

\begin{tabular}{lllc}
\hline Parameters & Classical values & Parameters & Classical values \\
\hline$N$ & 1000 & $\sigma_{y}$ & 14.522 \\
$n$ & 20 & $C_{x}$ & 0.042568 \\
$\bar{X}$ & 178.246 & $C_{y}$ & 0.170319 \\
$\bar{Y}$ & 82.817 & $\rho_{x y}$ & 0.024808 \\
$\sigma_{x}$ & 7.172 & $\beta_{2(x)}$ & -0.03362 \\
\hline
\end{tabular}

trosophic random variates (NRV) follows neutrosophic normal distributions (NND), i.e., $X_{N} \sim N N\left(\mu_{x N}, \sigma_{x N}^{2}\right) ; X_{N} \in$ $\left(X_{L}, X_{U}\right), \mu_{x N} \in\left(\mu_{x L}, \mu_{x U}\right), \sigma_{x N}^{2} \in\left(\sigma_{x L}^{2}, \sigma_{x U}^{2}\right)$ and $Y_{N} \sim N\left(\mu_{y N}, \sigma_{y N}^{2}\right) ; Y_{N} \in\left(Y_{L}, Y_{U}\right), \mu_{y N} \in\left(\mu_{y L}, \mu_{y U}\right)$, $\sigma_{y N}^{2} \in\left(\sigma_{y L}^{2}, \sigma_{y U}^{2}\right)$.

We took $Y_{N} \sim N N\left([76.0,84.9],\left[(12.9)^{2},(17.2)^{2}\right]\right)$, where $\mu_{y N} \in(76.0,84.9), \sigma_{y N} \in(12.9,17.2)$, and $X_{N} \sim N N\left([171.2,180.4],\left[(5.8)^{2},(6.7)^{2}\right]\right)$, where $\mu_{x N} \in$ (171.2, 180.4), $\sigma_{x N} \in(5.8,6.7)$ for simulating 1000 normal random variates. Table 4 shows the results of the neutrosophic data used to compare the performance efficiency of the proposed estimators and the traditional estimators under classical statistics. For classical statistics, Table 5 gives the information used to compute results.

Table 6 shows MSE under neutrosophic data and classical data and among all the estimators, $\bar{y}_{K N N}$ is more efficient under neutrosophic data with minimum MSE. When compare neutrosophic results with the classical, we may conclude that in situations, where data are not clear and crisp, instead of relying on a single value in the case of classical estimators, we have an interval to rely on for better results as we can accept the output if it falls in between these values, since we are dealing with uncertain or indeterminate data.

Table 7 includes the percentage relative efficiencies of neutrosophic estimators along with the classical estimator. It is clear from the results that $\bar{y}_{K N N}$ is the most efficient estimator whether it is neutrosophic data; or classical data. Furthermore, the neutrosophic estimators are more efficient than the classical estimators with low percentage relative efficiencies (PREs).
Table 6 Comparison of MSE of estimators

\begin{tabular}{llcc}
\hline SR. No & Estimator & MSE Neutrosophic & MSE Classical \\
\hline 1 & $\bar{y}_{K N N}$ & {$[8.403468$,} & 10.44198 \\
& & $14.98543]$ & \\
2 & $\bar{y}_{R N}$ & {$[8.639845$,} & 10.87109 \\
& & $15.4381]$ & \\
3 & $\bar{y}_{S D r N}$ & {$[8.639736$,} & 10.87085 \\
& & $15.4379]$ & \\
4 & $\bar{y}_{S K r N}$ & {$[8.639187$,} & 10.87029 \\
& & $15.43777]$ & \\
5 & $\bar{y}_{U S r N}$ & {$[8.63931$,} & 10.86943 \\
& & $15.43488]$ & \\
6 & $\bar{y}_{B T r N}$ & {$[8.444193$,} & 10.51607 \\
& & $15.08545]$ & \\
7 & $\bar{y}_{R r N}(a=0$, & {$[8.410288$,} & 10.45423 \\
& $b=0)$ & $14.98705]$ & \\
\multirow{2}{*}{8} & $\bar{y}_{\text {RrN }}(a=1, b=1)$ & {$[8.443529$,} & 10.51492 \\
& & $15.08421]$ & \\
\hline
\end{tabular}

Table 7 PREs of estimators (neutrosophic vs classical)

\begin{tabular}{llll}
\hline SR. No & Estimator & PREs & PREs Classical \\
& & Neutrosophic & \\
\hline 1 & $\bar{y}_{K N N}$ & {$[100,100]$} & 100 \\
2 & $\bar{y}_{R N}$ & {$[102.8128$,} & 104.1094 \\
& & $103.0207]$ & \\
3 & $\bar{y}_{S D r N}$ & {$[102.8115$,} & 104.1072 \\
& & $103.0194]$ & \\
4 & $\bar{y}_{S K r N}$ & {$[102.805$,} & 104.1018 \\
& & $103.0185]$ & \\
5 & $\bar{y}_{U S r N}$ & {$[102.8065$,} & 104.0936 \\
& & $102.9992]$ & \\
6 & $\bar{y}_{B T r N}$ & {$[100.4846$,} & 100.7096 \\
& & $100.6675]$ & \\
7 & $\bar{y}_{R r N}(a=0$, & {$[100.0811$,} & 100.1173 \\
& $b=0)$ & $100.0108]$ & \\
\multirow{2}{*}{8} & $\bar{y}_{R r N}(a=1$, & {$[100.4767$,} & 100.6985 \\
& $b=1)$ & $100.6592]$ & \\
\hline
\end{tabular}

\section{Discussion}

Tables 2 and 3 show the numerical results of MSE and PREs of the proposed neutrosophic ratio estimators for neutrosophic data from the population described in Table 1. It is observed from the indeterminacy interval results from Eq. (25) neutrosophic ratio-type estimator $\bar{y}_{\mathrm{KNN}}$, is highly efficient than the rest of the other proposed estimators under study in this article for the complete data. The indeterminacy interval results also indicate that the estimators $\bar{y}_{B T r N}$ and $\bar{y}_{R r N}$ for $(a=1$ and $b=1)$ are more efficient than all the other estimators except $\bar{y}_{\mathrm{KNN}}$ for the neutrosophic population that has a moderate and low (regardless of positive or negative direction) correlation between the study variable and the aux- 
iliary variable. The analysis by simulated neutrosophic data also verifies that the estimator $\bar{y}_{\mathrm{KNN}}$ is most efficient, while the estimators $\bar{y}_{B \operatorname{TrN}}$ and $\bar{y}_{R r n}(a=1, b=1)$ are precisely equally efficient. $\bar{y}_{R r N}(a=0, b=0)$ becomes a simple ratio estimator of mean, so it is better than others after $\bar{y}_{\mathrm{KNN}}$. The simulation results for both neutrosophic data and classical data, when compared, we conclude that the neutrosophic estimators give more reliable and more precise results, especially for unclear/ vague data. Neutrosophic results of MSEs in Table 6 suggested that our proposed neutrosophic estimator $\bar{y}_{K N N}$ with minimum MSE is better than other proposed estimators. PRE of estimator $\bar{y}_{\mathrm{KNN}}$ is also lowest among all results under neutrosophic data and classical data. All the estimators are unbiased (for order one), sufficient and consistent. In addition, the one with minimum variance is more efficient which is $\bar{y}_{\mathrm{KNN}}$ in this study.

\section{Conclusions}

The present study aims to use the ratio estimation method under neutrosophic data derived from simple random sampling. The study suggested that neutrosophic ratio-type estimators are more efficient than the classical estimators in the case of indeterminate data. Neutrosophic observations are of a unique form that comprises ambiguous, uncertain, or indeterminate values. The classical ratio estimation method provides single-valued results, which are sometimes not representative, especially in neutrosophic data. Through our proposed neutrosophic ratio-type estimators, we have tried to solve the issue of estimating the mean of the finite population in the case of neutrosophic data. This study is the first step, and a whole new area is open ahead for establishing improved estimators under different types of neutrosophic data under different sampling plans.

Acknowledgements The authors are deeply thankful to the editor and reviewers for their valuable suggestions to improve the quality of the paper.

\section{Declarations}

Conflict of interest No conflict of interest or competing interest is present among authors.

Open Access This article is licensed under a Creative Commons Attribution 4.0 International License, which permits use, sharing, adaptation, distribution and reproduction in any medium or format, as long as you give appropriate credit to the original author(s) and the source, provide a link to the Creative Commons licence, and indicate if changes were made. The images or other third party material in this article are included in the article's Creative Commons licence, unless indicated otherwise in a credit line to the material. If material is not included in the article's Creative Commons licence and your intended use is not permitted by statutory regulation or exceeds the permitted use, you will need to obtain permission directly from the copyright holder. To view a copy of this licence, visit http://creativecomm ons.org/licenses/by/4.0/.

\section{References}

1. Ali Z, Mahmood T (2020) Complex neutrosophic generalized dice similarity measures and their application to decision making. CAAI Trans Intelligence Technol 5. https://doi.org/10.1049/trit.2019.00 84

2. Aslam M (2018) A new sampling plan using neutrosophic process loss consideration. Symmetry 10(5):132. https://doi.org/10.3390/ sym 10050132

3. Aslam M (2019) Neutrosophic analysis of variance: application to university students. Complex Intell Syst 5:403-407. https://doi. org/10.1007/s40747-019-0107-2

4. Aslam M (2021) Monitoring the road traffic crashes using NEWMA chart and repetitive sampling. Int J Inj Contr Saf Promot 28(1):39-45. https://doi.org/10.1080/17457300.2020.183599 0

5. Aslam M (2021) A new goodness of fit test in the presence of uncertain parameters. Complex Intell Syst 7(1):359-365. https:// doi.org/10.1007/s40747-020-00214-8

6. Aslam M (2021) A study on skewness and kurtosis estimators of wind speed distribution under indeterminacy. Theoret Appl Climatol 143(3):1227-1234. https://doi.org/10.1007/s00704-020-035 09-5

7. Aslam M, Arif O, Khan R (2020) New diagnosis test under the neutrosophic statistics: an application to diabetic patients. Biomed Res Int 00:7. https://doi.org/10.1155/2020/2086185

8. Bahl S, Tuteja RK (1991) Ratio and product type exponential estimators. J Inf Optim Sci 12(1):159-164. https://doi.org/10.1080/02 522667.1991.10699058

9. Chakraborty A, Banik B, Mondal S, Alam S (2020) Arithmetic and geometric operators of pentagonal neutrosophic number and its application in mobile communication service based MCGDM problem. 32:61-79. 10.5281/zenodo.372314

10. Chakraborty A, Mondal S, Alam S, Mahata A (2020) Cylindrical neutrosophic single-valued numberand its application in networking problem. Multi Criterion Decision Making Problem Graph Theory 5:68-77. https://doi.org/10.1049/trit.2019.0083

11. Chakraborty A, Mondal SP, Alam S, Dey A (2021) Classification of trapezoidal bipolar neutrosophic number, de-bipolarization technique and its execution in cloud service-based MCGDM problem. Complex Intell Syst 7(1):145-162. https://doi.org/10.1007/s4074 7-020-00170-3

12. Chen J, Ye J, Du S (2017) Scale effect and anisotropy analyzed for neutrosophic numbers of rock joint roughness coefficient based on neutrosophic statistics. Symmetry 9(10):208. https://doi.org/10.33 90/sym9100208

13. Cochran WG (1940) The estimation of the yields of cereal experiments by sampling for the ratio of grain to total produce. J Agric Sci 30(2):262-275. https://doi.org/10.1017/S0021859600048012

14. Cochran WG (1977). Sampling Techniques (Third Edition ed.), \& Sons, New York

15. Custom Weather. (2020). Retrieved from https://www.timeanddate. com/weather/pakistan/lahore/historic

16. Haque T, Chakraborty A, Mondal S, Alam S (2020) A new approach to solve multi-criteria group decision making problems by exponential operational law in generalised spherical fuzzy environment. 5:106-114. https://doi.org/10.1049/trit.2019.0078

17. Jan N, Zedam L, Mahmood T, Ullah K, Ali Z (2019) Multiple attribute decision making method under linguistic cubic informa- 
tion. J Intell Fuzzy Syst 36:253-269. https://doi.org/10.3233/JIFS181253

18. Kadilar C, Cingi H (2006) An improvement in estimating the population mean by using the correlation coefficient. Hacettepe J Math Stat 35(1):103-109

19. Khan H, Sanaullah A, Amjad M, Siddiqi A (2014) Improved exponential ratio type estimators for estimating population mean regarding full information in survey sampling. World Appl Sci J 26(5):1897-1902

20. Li D-F, Mahmood T, Ali Z, Dong Y (2020) Decision making based on interval-valued complex single-valued neutrosophic hesitant fuzzy generalized hybrid weighted averaging operators. J Intell Fuzzy Syst 38:4359-4401. https://doi.org/10.3233/JIFS-191005

21. Liu P, Ali Z, Mahmood T (2020) The distance measures and cross-entropy based on complex fuzzy sets and their application in decision making. J Intell Fuzzy Syst 39:1-24. https://doi.org/1 0.3233/JIFS-191718

22. Pandey BN, Dubey V (1988) Modified product estimator using coefficient of variation of auxiliary variate. Assam Stat Rev 2(2):64-66

23. Robson DS (1957) Applications of multivariate polykays to the theory of unbiased ratio-type estimation. J Am Stat Assoc 52(280):511-522. https://doi.org/10.1080/01621459.1957.105014 07

24. Singh HP, Kakran MS (1993) A modified ratio estimator using known coefficient of kurtosis of an auxiliary character. J Indian Soc Agric Stat

25. Singh HP, Tailor R (2003) Use of known correlation coefficient in estimating the finite population mean. Stat Trans 6(4):555-560
26. Singh R, Chauhan P, Sawan N, Smarandache F (2007) Auxiliary information and a priori values in construction of improved estimators: Infinite Study.

27. Singh R, Kumar M, Chaudhary M, Smarandache F (2009) Estimation of mean in presence of non-response using exponential estimator. Infinite Study

28. Sisodia BVS, Dwivedi VK (1981) Modified ratio estimator using coefficient of variation of auxiliary variable. J Indian Soc Agric Stat 33:13-18

29. Smarandache F (1998) Neutrosophy: neutrosophic probability, set, and logic: analytic synthesis \& synthetic analysis.: American Research Press.

30. Smarandache F (2014) Introduction to neutrosophic statistics: Sitech \& Education Publishing

31. Upadhyaya LN, Singh HP (1999) Use of transformed auxiliary variable in estimating the finite population mean. Biometrical $\mathbf{J}$ 41(5):627-636. https://doi.org/10.1002/(SICI)1521-4036(199909 )41:5\%3c627::AID-BIMJ627\%3e3.0.CO;2-W

32. Upadhyaya LN, Singh HP, Chatterjee S, Yadav R (2011) Improved ratio and product exponential type estimators. J Stat Theory Pract 5(2):285-302. https://doi.org/10.1080/15598608.2011.10412029

Publisher's Note Springer Nature remains neutral with regard to jurisdictional claims in published maps and institutional affiliations. 